\title{
AN EVALUATION OF THE EFFECTS OF A FUNCTIONAL ENERGY DRINK ON POST-LUNCH AND EARLY EVENING DRIVING PERFORMANCE
}

\author{
A.M. Parkes, B.F. Sexton, S. Burton, \\ Transport Research Laboratory, Crowthorne, Berkshire, England. \\ E-mail:aparkes@trl.co.uk \\ H.L. Hu, J.A. Shaw and B.P. Daggy \\ GlaxoSmithKline, Nutritional Healthcare R\&D, Slough, England
}

\begin{abstract}
Summary: This paper reports the results of a pilot study designed to evaluate the effect of an energy drink on mental performance and driving. 24 healthy subjects were tested after consumption of a placebo or an energy drink in a double-blind crossover study. Measures included a laboratory test of Adaptive Tracking (AT), and a simulated drive involved a $40 \mathrm{Km}$ motorway route in an advanced motion-based simulator. Self-report scales of sleepiness revealed a significant difference between placebo and energy drink. Though both drinks provided an alerting effect, both the level and duration of the effect observed after consumption of the energy drink was greater. Performance on the AT task was significantly improved. This improvement in hand-eye coordination was reflected in better lane-keeping performance in the simulated driving task. There was also a consistent tendency when the drivers drank placebo to drive slightly faster in traffic than when drinking the energy drink. These preliminary findings, which demonstrate that consumption of even a relatively small volume $(250 \mathrm{ml})$ of an energy drink can have an effect on sleepiness, lane keeping and speed choice in simulated traffic, could have implications for future highway safety.
\end{abstract}

\section{INTRODUCTION}

Energy drinks may have a contribution to make to road safety by either delaying the onset of fatigue or reducing its effects during driving. In-depth accident studies suggest that sleep may be a contributing factor in between 6 and 30 percent of road injuries, depending on a range of factors including type of road, time-of-day and severity of the accident (Maycock 1996). This paper reports the results of a pilot study that used methods originally developed to study driver impairment and driving performance as a function of medical or social drugs use. The focus was not on detailed analysis of the physiological response of the driver, but instead the study sought to build up a rich picture of the behaviour, and include subjective measures of fatigue and situation awareness.

There are obvious safety and ethical considerations associated with performing experiments of this type on real roads, whether public highways or private road networks. For this reason, in this initial study, driving simulation offered the only realistic option. This approach provided a number of additional benefits, including: 
- Repeatability and control of experimental protocol

- Detailed and accurate logging of driver behaviour

- Detailed and accurate logging of vehicle control parameters

\section{METHOD}

24 participants (12 male, 12 female: Mean age 36.21, SD 6.75) were tested after consumption of a placebo or energy drink in a double-blind crossover study. Before inclusion in the main trial, subjects attended a screening visit where it was determined that they were healthy, of normal build (BMI Mean 25.08, SD 2.44), with no sleep problems or excessive daytime sleepiness. They were non-smokers, free of medication and modest consumers of alcohol. None took regular naps. They were also screened on the 'morningness-eveningness' scale (Horne and Ostberg, 1976), and were 'neither' types.

The energy drink (Solstis ${ }^{\circledR}$, GlaxoSmithKline, Brentford UK) provided 75 mg caffeine and 37.5 g glucose; the placebo was matched for colour, temperature and taste, and both were administered as a single $250 \mathrm{ml}$ serving. Each subject was tested after a normal restful night's sleep, in a balanced order on four separate occasions, at one-week intervals. Two levels of fatigue were incorporated by testing subjects during different stages of the diurnal pattern; during the post-lunch dip where alertness is at a low point, and again in the early evening when alertness is higher. In each situation either an energy drink or a placebo was administered. Each participant was tested four times (Time-of-day x 2, Drink x 2). One hour before each session, the participant had a light sandwich meal without alcohol or caffeine.

The TRL Driving Simulator consists of a medium sized saloon car surrounded by large projection screens giving 210-degree horizontal and 40-degree vertical front vision, and 60degree horizontal and up to 40-degree vertical rear vision, enabling the normal use of all vehicle mirrors. The road images were generated by advanced graphic workstations and projected at life size onto the screens. The car body shell incorporates hydraulic rams that supply motion to simulate the heave, pitch and roll experienced in normal braking, accelerating and cornering. Also, when negotiating curves, the simulator provides realistic forces experienced by the driver through the steering wheel. The realism of the driving experience is further enhanced by the provision of car engine noise, external road noise, and the sounds of passing traffic.

The study required a realistic but relatively undemanding road environment. We did not wish to raise the subjects' activation levels artificially by introducing race-track or demanding urban environments. Therefore, a 40-kilometre motorway scenario with moderate traffic flow was chosen. The motorway drive had two stages. In the first, the driver engaged with other traffic in a normal fashion, and was allowed to choose speed, lane, distance from other vehicles, and so on. During this stage of the drive (around 20 minutes), the subject self-reported the level fatigue, and also answered probe questions of situation awareness (after Endsley 1987). This was measured at a point in moderate traffic by blanking the simulation without warning the driver, so that the scene was no longer available for visual inspection, and asking a series of probe questions about the presence and actions of surrounding vehicles. Measures were also taken of speed selection, reaction times and minimum-time-headways to lead vehicles in busy traffic. In the second stage of the motorway, other traffic was removed and the ability to maintain a 
constant speed of exactly $35 \mathrm{mph}(56.33 \mathrm{kph})$ and central lane position during a route of curves of varying radius was recorded.

Table 1 describes the study sequence. Individual participant sessions lasted for around 150 minutes, including 30 minutes prior to consumption of the drinks and 120 afterwards.

At seven suitable points during the session (three pre-drink) the subject was required to complete the Karolinska Sleepiness Scale (KSS) (Arkerstedt \& Gillberg 1989), as a self-report measure of fatigue. KSS is a nine-point scale, with semantic anchors [1 = 'extremely alert', 5 = 'neither alert nor sleepy', 9 = ‘extremely sleepy - fighting sleep’]

Adaptive tracking, which is a measure of hand eye co-ordination, was measured twice; once before and once after consumption of the drinks. It involved a joystick-controlled twodimensional pursuit tracking task, displayed on a large CRT, the degree of difficulty of which is continuously modified by performance feedback. It was first described in Borland and Nicholson (1974) and has been extensively used in studies of drugs and alcohol.

Useful Field of View (UFOV) is a PC based test that determines the reduction in the ability to correctly identify a central and peripheral target, once those targets are presented against a field of distracting objects.

Table 1. Study Sequence.

\begin{tabular}{|c|l|c|l|}
\hline $\begin{array}{c}\text { Minutes } \\
\text { from } \\
\text { arrival }\end{array}$ & \multicolumn{1}{|c|}{ Activity } & $\begin{array}{c}\text { Minutes } \\
\text { from } \\
\text { arrival }\end{array}$ & \multicolumn{1}{|c|}{ Activity } \\
\hline 0 & Arrival & 65 & KSS \\
\hline 5 & $\begin{array}{l}\text { Questionnaire re sleep and food } \\
\text { during preceding 24 hours }\end{array}$ & 70 & $\begin{array}{l}\text { Start Driving Simulator trial } \\
\text { (30 minutes) }\end{array}$ \\
\hline 7 & $\begin{array}{l}\text { Karolinska Sleepiness Scale } \\
\text { (KSS) }\end{array}$ & $(85)$ & $\begin{array}{l}\text { During drive, KSS followed } \\
\text { by Situation Awareness }\end{array}$ \\
\hline 10 & Baseline Simulator drive & 100 & KSS \\
\hline 20 & KSS & 110 & Adaptive Tracking \\
\hline 30 & Adaptive Tracking & 115 & Useful field of View (UFOV) \\
\hline 35 & KSS & 145 & KSS \\
\hline $\mathbf{4 0}$ & Drink & 150 & Refreshment \& home \\
\hline
\end{tabular}

\section{RESULTS}

The analysis used SAS and a hierarchic model; fitting the participant effect, then visit effect, followed by drink and time of day. There were no carry-over effects revealed. For brevity, only significant results are reported in detail.

\section{Karolinska Self Report Scale of Sleepiness (KSS)}

There was an increasing trend in reported sleepiness in the 30 minutes preceding the drink.

Compared to the placebo, consumption of the energy drink led to lower scores in both afternoon and evening sessions $(\mathrm{t}=2.16, \mathrm{p}<.05)$. Consumption of both drinks clearly had a refreshing effect, 
but the effect of the energy drink was greater and sustained longer. After 1.5 hours, the placebo scores were higher than before the drink, showing increased fatigue. The energy drink scores were at a significantly lower level $(\mathrm{t}=3.44, \mathrm{p}<.01)$.

Table 2. Mean KSS scores at seven points through the 150 minute session.

\begin{tabular}{|l|l|l|l|l|l|l|l|l|}
\hline $\begin{array}{l}\text { Before drink } \\
\text { measure }\end{array}$ & $\mathbf{1}$ & $\mathbf{2}$ & $\mathbf{3}$ & $\begin{array}{l}\text { Post drink } \\
\text { measure }\end{array}$ & $\mathbf{4}$ & $\mathbf{5}$ & $\mathbf{6}$ & $\mathbf{7}$ \\
\hline $\begin{array}{l}\text { Time(min)from } \\
\text { start of session }\end{array}$ & 7 & 20 & 35 & & 65 & 85 & 100 & 145 \\
\hline & & & & & & & & \\
\hline Afternoon & 3.63 & 3.79 & 4.73 & Placebo & 4.17 & 3.88 & 4.21 & 5.14 \\
\hline & & & & Energy Drink & 4.04 & 3.71 & 3.96 & 4.25 \\
\hline Evening & 4.27 & 4.32 & 5.23 & Placebo & 4.50 & 4.38 & 4.29 & 5.21 \\
\hline & & & & Energy Drink & 4.50 & 4.04 & 4.48 & 4.77 \\
\hline
\end{tabular}

It is also important to note that the fifth reading was taken during the simulated drive, and was nearly a statistically significantly lower sleepiness score than the placebo $(t=1.63, p=0.054)$.

\section{Adaptive Tracking}

Table 3 Mean Adaptive Tracking speed (m/s)

\begin{tabular}{|l|l|l|l|}
\hline & Pre drink & Placebo & Energy Drink \\
\hline Afternoon & 27.63 & 28.48 & 30.55 \\
\hline Evening & 26.61 & 29.33 & 29.77 \\
\hline
\end{tabular}

The mean scores represent tracking speed in metres per second, so higher values represent better performance. After consumption of the energy drink in the afternoon session, subjects showed significantly higher adaptive tracking scores than after consumption of the placebo $(\mathrm{t}=2.33$, $\mathrm{p}<.05$ ). Compared with the pre-drink baseline, consumption of the energy drink improved scores by 10 percent in the post lunch dip period $(\mathrm{t}=4.26, \mathrm{p}<.01)$. Combining afternoon and evening sessions, the AT speed was significantly faster in the energy drink groups than the pre-drink speed $(\mathrm{t}=4.29$, and $\mathrm{t}=6.48$ for placebo and energy drink respectively, both with $\mathrm{p}<.001)$.

\section{Speed Choice in Traffic}

In the afternoon sessions subjects drove significantly faster after consumption of the placebo, $66.5 \mathrm{mph}$ for the placebo as compared to $64.3 \mathrm{mph}$ for the energy drink, (mean difference $=2.23$ $\mathrm{mph}, \mathrm{t}=1.87, \mathrm{p}<.05$ ). No significant difference was found in the evening sessions though the trend was repeated.

\section{Driving Accuracy Measures}

The standard deviation of lane position was calculated during the curved section of the motorway. There was a general trend for reduced variation after drivers had consumed the energy drink, (mean difference $=0.148 \mathrm{~m}, \mathrm{t}=1.168, \mathrm{p}=.059$ ). There was no significant difference between groups in terms of ability to maintain the prescribed speed. 


\section{DISCUSSION}

Given that this was a preliminary study, using an approach geared to assessing gross impairment due to medical and social drug usage, it is not surprising that some tests were better able to discriminate between the drinks than others. Subjective self-report of fatigue state was the clearest measure, and results are in line with those previously reported (Smit \& Rogers 2000). Though both drinks provided an alerting effect, both the level and duration of the effect observed after energy drink consumption was greater.

Performance on the Adaptive Tracking task was significantly better after consumption of the energy drink than after the placebo. This improvement in hand-eye coordination was reflected in better lane keeping performance in the simulated driving task, though the result did not quite achieve statistical significance

There was also a consistent tendency when the subjects drank the placebo for them to drive faster in traffic than after drinking the energy drink. Further research is needed to determine if this effect can be replicated; and whether the underlying mechanism is a wish to complete the task quicker, or to seek extra stimulation from the drive to counteract the fatigue, or due to a reduction in the basic ability to interpret speed when more tired. However, the findings on speed and reactions to other traffic were completely consistent with those of Stein (1995). He had drivers given instructions to drive with a threat of a penalty for driving $3 \mathrm{mph}$ over a specified limit, in a driving simulator. He found that fatigued drivers were less able to avoid penalties as their speed tended to drift above the limit, though coarser measures of performance such as collisions with other traffic did not show any differences.

Other measures failed to discriminate between the drinks (UFOV, Situation Awareness, driving at a set speed, and reactions to other vehicles in traffic). These point to a need to increase sensitivity if these are to be used successfully in this type of research, where reasonably subtle effects are expected.

These preliminary findings demonstrate that even a small volume $(250 \mathrm{ml})$ of a specially formulated energy drink can have a consistent effect on sleepiness, lane keeping and speed choice in traffic. These findings are important, and if supported by further research could have implications for future highway safety.

\section{REFERENCES}

Akerstedt \& Gillberg, (1989). Subjective and Objective Sleepiness in the Active Individual, Int. J. Neuroscience, 1990, Vol. 52, pp 29-37.

Borland, RG \& Nichilson, AN (1974). Human performance after a barbituate. Br. J. of Clin. Pharmacology. 1, 209-215.

Endsley, M. R. (1987). SAGAT; A methodology for the measurement of situation awareness (NOR DOC 87-83). Hawthorne, CA: Northrop Corporation.

Horne J A \& Ostberg O. (1976). A self assessment questionnaire to determine morningnesseveningness in human circadian rythmns. Int. J. of Chrono-biology, Vol. 4, 97-110

Maycock, G. (1996). Sleepiness and driving: the experience of UK car drivers. J. Sleep Res. 5, 229-237. 
Smit H J \& Rogers P J. (2000). Effects of low doses of caffeine on cognitive performance, mood and thirst in low and high caffeine consumers. Psychopharmacology, 152: 167173.

Stein, AC. (1995) Detecting fatigued drivers with vehicle simulators. In L. Hartley (ed) Fatigue and Driving. Taylor \& Francis, London. 